\title{
4 O OLHAR DA FAMÍlLIA SOBRE O MANEJO DE UM HOSPITAL DIA EM SAÚDE MENTAL
}

\author{
| César Augusto Trinta Weber ${ }^{1}$; Mario Francisco Juruena ${ }^{2} \mid$
}

\section{RESUMO}

CONTEXTO: No tratamento das pessoas com transtorno mental é fundamental o apoio da família e da comunidade. De um lado, as terapias de suporte à família para a compreensão do fenômeno do adoecimento, desmistificando a doença e as suas formas de tratamento e, de outro, os mecanismos de estímulo às redes sociais de apoio, como formas de amparo na perspectiva de um novo olhar sobre o cuidado em saúde mental.

OBJETIVO: Investigar a percepção da família sobre as estratégias terapêuticas de um hospital dia em saúde mental para a inclusão de seus usuários.

MÉTODO: Estudo qualitativo, pesquisa documental e estudo de caso.

RESULTADOS: Os familiares demonstraram possuírem alguns conhecimentos sobre a doença mental. Os aprendizados teóricos e práticos adquiridos no hospital dia vêm permitindo-lhes compreender e lidar melhor com o fenômeno do adoecimento que vivenciam com os seus parentes. À medida que a família começa a fazer parte do processo de cuidado, além de comprometerem-se com as atividades terapêuticas propostas, esta passa a reconhecer os efeitos de cada etapa desse tratamento. Em muitos casos, os primeiros resultados passam a ser percebidos pela resposta positiva na melhora do quadro clínico e reintegração social. Sobre as Redes Sociais de Apoio as pessoas com transtornos mentais e suas famílias, a maioria dos participantes referiram desconhecê-las.

CONCLUSÕES: Os aprendizados teóricos e práticos acumulados pela família sobre a doença mental permitiram-lhes compreender e lidar melhor com o fenômeno do adoecimento.

PALAVRAS-CHAVE: Hospital dia; Psiquiatria comunitária; Saúde mental

\section{RESUMEN}

"La mirada de la familia en la gestión de un centro de atención diurna de salud mental“

CONTEXTO: En el tratamiento de los enfermos mentales es esencial para mantener a la familia y la comunidad. Por un lado, las terapias de apoyo a la familia para la comprensión de la enfermedad del fenómeno, desmitificar la enfermedad y sus formas de tratamiento y, por el otro, los mecanismos de incentivo a las redes de apoyo social, como formas de apoyo en la perspectiva de una nueva mirada a la atención de salud mental.

OBJETIVO: Investigar la percepción de las estrategias terapéuticas de un hospital de día de salud mental para la inclusión de sus miembros de la familia.

MÉTODO: Estudio cualitativo, la investigación documental y estudio de casos.

RESULTADOS: La familia demostró tener algún conocimiento sobre la enfermedad mental. Los aprendizajes teóricos y prácticos adquiridos en el centro de atención diurna les ha permitido comprender mejor y afrontar el fenómeno de la enfermedad que la experiencia con sus familiares. A medida que la familia se convierte en parte del proceso de atención, y comprometerse con las actividades terapéuticas propuestas, se va a reconocer los efectos de cada paso de este tratamiento. En muchos casos, los primeros resultados empiezan a ser percibidos por la respuesta positiva en la mejora clínica y la reintegración social. Sobre la gente de apoyo de una red social con trastornos mentales y sus familias, la mayoría de los participantes informaron desconocido ellos.

CONCLUSIONES: Los aprendizajes teóricos y prácticos acumulados por la familia sobre la enfermedad mental que les permitió comprender mejor y hacer frente a la enfermedad del fenómeno.

DESCRIPTORES: Centro de atención diurna; Psiquiatría comunitaria; Salud mental

\section{ABSTRACT \\ "Family's view on the therapeutic of a mental health partial hos- pitalization"”}

BACKGROUND: In the treatment of mental patients is essential to support the family and the community. On the one hand, the family-support therapies for understanding the illness of the phenomenon, demystifying the disease and its forms of treatment and on the other, the incentive mechanisms to social support networks, as forms of support in the perspective of a new look at the mental health care.

AIM: To investigate the family's perception of the therapeutic strategies of a day hospital for mental health for the inclusion of its members.

METHODS: Qualitative study, documentary research and case study.

RESULTS: The family demonstrated having some knowledge about mental illness. The theoretical and practical learnings acquired in the partial hospitalization has allowed them to better understand and cope with the illness phenomenon that experience with their relatives. As the family becomes part of the care process, and commit to the therapeutic activities proposed, it goes to recognize the effects of each step of this treatment. In many cases, the first results start to be perceived by the positive response in clinical improvement and social reintegration. About Social Networking Support people with mental disorders and their families, most participants reported unknow them.

CONCLUSIONS: The theoretical and practical learnings accumulated by the family about mental illness allowed them to better understand and cope with the illness of the phenomenon

\section{KEYWORDS: Day care; Medical; Community psychiatry; Men- tal health}

Submetido em 13-09-2016

Aceite em 30-03-2017

1 César Augusto Trinta Weber, MD. MSc. PhD. Avenida Ecoville, 190, casa 07. Bairro Sarandi. Porto Alegre/RS/Brasil. CEP: 91150-400, trintaweber@hotmail.com 2 Mario Francisco Juruena, MD, MSc, Dip CBT, MPhil, PhD. Avenida Tenente Catão Roxo, 2650, Ribeirão Preto/SP/Brasil: CEP 14051-140, juruena@fmrp.usp.br 


\section{INTRODUÇÃO}

As pessoas com transtornos mentais, a exemplo de pessoas com transtornos de outra natureza, apresentam um grau maior ou menor de incapacidade para determinadas atividades de vida diária. Para fins da construção e usufruto das políticas públicas afirmativas, são classificados como pessoas portadoras de deficiência e, portanto, com necessidades especiais (Weber, 2012).

Com efeito, os termos pessoa com transtorno mental e pessoa com deficiência utilizados neste texto buscam, por não serem sinônimos, alcançarem o propósito de aproximação dos seus significados em seu ponto de contato, ao simbolizarem aquelas pessoas que apresentam necessidades próprias e diferentes, as quais requerem uma atenção específica, no caso, as pessoas que apresentam significativas diferenças sensoriais ou intelectuais, decorrentes de fatores inatos ou adquiridos e que acarretam um próprio modo de pensar, sentir e se comportar no mundo, muitas vezes com importantes prejuízos familiares, sociais e laborais.

Até bem pouco tempo, uma pessoa portadora de deficiência era entendida como sendo aquela que apresenta, em caráter permanente, perdas ou anormalidades de sua estrutura ou função psicológica, fisiológica ou anatômica, que gerem incapacidade para o desempenho das atividades dentro do padrão considerado normal para o ser humano.

Atualmente, este conceito vem sendo repensado à luz da estreita relação existente entre as limitações que experimentam as pessoas portadoras de deficiências, a concepção e a estrutura do meio ambiente e a atitude da população em geral com relação à questão.

$\mathrm{Na}$ raiz dessa nova abordagem está a inclusão social, entendida como o processo pelo qual a sociedade se adapta para incluir, em seus sistemas sociais gerais, pessoas com necessidades especiais e, simultaneamente, estas se preparam para assumir seus papéis na sociedade. A inclusão social constitui, então, um processo bilateral no qual as pessoas, ainda excluídas, e a sociedade buscam, em parceria, equacionar problemas, decidir sobre soluções e efetivar a equiparação de oportunidades para todos (Weber, 2012). Segundo o Ministério da Saúde do Brasil (MS), a Política Nacional de Saúde Mental (PNSM), apoiada na Lei 10.216/2001, busca consolidar um modelo de atenção à saúde mental aberto e de base comunitária. Isto é, que garante a livre circulação das pessoas com transtornos mentais pelos serviços, comunidade e cidade, e oferece cuidados com base nos recursos que a comunidade oferece.
Este modelo conta com uma rede de serviços e equipamentos variados, entre esses, a atenção em hospital dia que na assistência em saúde mental representa um recurso intermediário entre a internação e o ambulatório, que desenvolve programas de atenção de cuidados intensivos por equipe multiprofissional, visando substituir a internação integral.

O hospital dia do Hospital das Clínicas da Faculdade de Medicina de Ribeirão Preto da Universidade de São Paulo (HD/HCFMRP/USP) é um dos equipamentos terapêuticos que atende ao modelo de assistência à saúde mental preconizado pela Reforma da Assistência Psiquiátrica.

No tratamento das pessoas com transtorno mental é fundamental o apoio da família e da comunidade. De um lado, as terapias de suporte à família para a compreensão do fenômeno do adoecimento, desmistificando a doença e as suas formas de tratamento e, de outro, os mecanismos de estímulo às redes sociais de apoio, como formas de amparo como um novo olhar sobre o cuidado em saúde mental (Weber \& Juruena, 2014). Este artigo foi desenvolvido com base nos resultados de estágio de pós-doutoramento cuja investigação teve como objetivo geral o de investigar a percepção da família sobre as estratégias terapêuticas instituídas pelo HD/HCFMRP/USP para a inclusão de seus usuários.

\section{MÉTODO}

Estudo qualitativo aprovado pelo Comitê de Ética em Pesquisa do Hospital das Clínicas da Faculdade de Medicina de Ribeirão Preto da Universidade de São Paulo (Parecer $n^{\circ}$ 829.098). Pesquisa descritiva exploratória, estudo de caso. Foram utilizadas as técnicas e procedimentos de revisão documental, da observação participante, entrevistas semi-estruturadas em profundidade. As atividades de campo para coleta dos dados e observação participante foram realizadas no período de 31 de março a 17 de abril de 2014, e se desenvolveram no horário das $7 \mathrm{~h}$ às $18 \mathrm{~h}$, nas dependências do $\mathrm{HD} /$ HCFMRP/USP, localizado no Campus Universitário, em Ribeirão Preto, São Paulo, Brasil. Neste período foram revisados os prontuários médicos de todos os pacientes que se encontravam em regime de semi-internação no HD/HCFMRP/USP e realizadas 15 entrevistas semi-estruturadas com os familiares desses pacientes, após os mesmos terem concordado com a participação neste estudo e de terem lido, compreendido e assinado o Termo de Consentimento Livre e Esclarecido. 
As entrevistas foram gravadas em meio digital e, depois, transcritas para análise das categorias de estudo identificadas pelo pesquisador. A pesquisa foi realizada tendo como garantia as normas éticas e confidenciais.

\section{RESULTADOS}

A Tabela 1 apresenta o grau de parentesco, idade, sexo e município de origem dos familiares dos usuários do HD participantes desta investigação. O grau de parentesco e a idade, obtidos por auto-relato, foram aceitos como verdadeiros sem a exigência de comprovação documental. Para garantia do anonimato os familiares foram identificados pela letra $\mathrm{P}$ seguida de numeração crescente, em obediência a ordem das datas de realização de cada entrevista.

Tabela 1 - Grau de Parentesco, Idade, Sexo e Município de Origem dos Familiares Participantes

\begin{tabular}{|c|c|c|c|c|}
\hline Participantes & $\begin{array}{c}\text { Grau de } \\
\text { Parentesco }\end{array}$ & Idade & Sexo & $\begin{array}{c}\text { Município de } \\
\text { Origem }\end{array}$ \\
\hline P1 & Mãe & 54 & F & Serra azul \\
\hline P2 & Irmã & 69 & F & Ribeirão Preto \\
\hline P3 & Esposo & 30 & M & Ribeirão Preto \\
\hline P4 & Esposo & 30 & M & Jardinópolis \\
\hline P5 & Filha & 24 & F & Ribeirão Preto \\
\hline P6 & Mãe & 60 & F & Guariba \\
\hline P7 & Esposo & 47 & M & Ribeirão Preto \\
\hline P8 & Esposa & 52 & F & Ribeirão Preto \\
\hline P9 & Pai & 71 & M & Ribeirão Preto \\
\hline P10 & Primo & 47 & M & Jardinópolis \\
\hline P11 & Esposa & 42 & F & Pradópolis \\
\hline P12 & Mãe & 58 & F & Cravinhos \\
\hline P13 & Esposo & 67 & M & Ribeirão Preto \\
\hline P14 & Irmã de & 53 & F & Pitangueiras \\
\hline P15 & Espoşão & & & \\
\hline & & 39 & M & Sertãozinho \\
\hline
\end{tabular}

As categorias temáticas que emergiram após a análise das narrativas dos familiares participantes foram: Estratégias para fornecer informações aos pacientes e familiares sobre a natureza e o tratamento da doença; Percepções dos familiares sobre as terapêuticas de cuidado praticadas no HD/HCFMRP/USP em seu cotidiano; Grupo Comunitário e Redes Sociais de Apoio.
Estratégias para Fornecer Informações aos Pacientes e Familiares sobre a Natureza e o Tratamento da Doença

As implicações da categoria temática "Estratégias para fornecer informações aos pacientes e familiares sobre a natureza e o tratamento da doença" serão analisadas por meio de duas subcategorias: Aprendizados teóricos e práticos para compreender e lidar melhor com o fenômeno do adoecimento e Sentimentos vivenciados pela experiência de ser familiar de uma pessoa com transtorno mental.

\section{Aprendizados Teóricos e Práticos para Compreender e Lidar Melhor com o Fenômeno o Adoecimento}

Os participantes quando perguntados sobre o que está acontecendo com o seu parente em tratamento no HD/ HCFMRP/USP, de onde vem e como se manifesta a doença que o acomete, responderam demonstrando que possuem alguns conhecimentos - ainda que não sistematizados e de natureza empírica - como revelam essas falas:

(...) só sei que ela escuta uma "voz". Ela se machuca, a voz manda ela se jogar debaixo de ônibus, a voz manda ela se jogar debaixo de qualquer coisa, se corta, se arranha toda a barriga. [P1]

(...) pegou do parto da menina. (...) já veio de quando ela ganhou a menina. (...) ela desceu na cama da operação, que ela operou para ter a menina, e já caiu dentro do banheiro. Depois teve a recaída da dieta e já ficou desse jeito. Veio da gravidez. [P6]

(...) acho que é muita tristeza, de tudo o que aconteceu, que nem um tio matou o irmão dele dentro de casa, e isso mexeu muito com ele. De criança o pai bebia muito, judiava da mãe, ele sofria muito com isso. Ele se isola de tudo, só quer dormir, ficar trancado. Não quer sair mais. Uma doença que ele não tem vontade de fazer mais nada. [P11]

(...) esse problema é porque ela sempre foi uma menina mais rebelde. Então ela falava: "vocês sempre me bateram mais". Ela sempre teve um problema porque ela achava que não era filha da gente. [P12]

Entre as abordagens terapêuticas desenvolvidas no HD/HCFMRP/USP os familiares mencionaram que os aprendizados teóricos e práticos sobre a doença mental vêm permitindo-lhes compreender e lidar melhor com o fenômeno do adoecimento que vivenciam com os seus parentes. 
As informações acumuladas pelas suas participações nas atividades de tratamento, as quais envolvem, entre outras, noções mais rigorosas sobre como se manifestam e quais os tratamentos possíveis para os diversos transtornos mentais, tornam possível a aceitação com maior naturalidade da realidade de tais doenças, como se pode observar nessas narrativas:

(...) o hospital dia é o tratamento da doença mental, de uma forma ampla, não apenas medicamentoso. (...) oferece tratamento terapêutico que disponibiliza através dos diversos profissionais: terapia ocupacional, psicólogo, psiquiatras, e todos os profissionais que trabalham nessa área. (...) E pelo menos recolocar a pessoa num eixo aceitável de uma vida comum. [P3]

(...) é antes a gente achava que não era muito grave né, que era uma doença assim que a gente achava que nem precisava de tratamento. (...) No começo nós falávamos que era uma frescura (...) Agora estamos entendo a doença dela e do que ela precisa para melhorar. [P4] (...) tem ajudado nós a se relacionar com ela também, porque a gente não tinha noção de como lidar, como conversar. Se ela não quer conversar agora depois tentar conversar mais tarde. (...) Aprendemos a forma de lidar também com isso. [P5]

\section{Sentimentos Vivenciados pela Experiência de Ser Fa- miliar de Uma Pessoa com Transtorno Mental}

O significado da doença mental para os familiares participantes, foi referido pelos sentimentos de tristeza, isolamento, desilusão e de perda que experimentam no dia-a-dia de convivência tão próxima com uma pessoa com transtorno mental, como se constata nesses relatos:

(...) eu sei que é triste, viu doutor, é triste. Ela fica só dentro do quarto quando ela tá em casa. [P1]

(...) é bem desconfortável para a família né? Tem uma pessoa que parece presa no mundo dela, não consegue ter uma vida social normal como outra pessoa. Aparentemente não parece nem doença, porque a pessoa não tem dor, um sintoma assim provável, um exame né? [P10]

(...) é um sentimento de perda. Porque você perder as coisas naturais da vida, com o envelhecimento, isso é natural, faz parte. Mas de repente você está com a sua mulher e ela sai da sua vida, a gente não consegue mais conviver, é tristeza constante, desequilibra todo o jeito de ser. É uma perda antecipada. [P13]
Percepções dos Familiares sobre as Terapêuticas de Cuidado no HD/HCFMRP/USP em seu Cotidiano

À medida que a família começa a fazer parte do processo de cuidado, comprometendo-se com as atividades terapêuticas propostas, esta passa a reconhecer e distinguir os vários itinerários terapêuticos percorridos durante a semi-internação no HD/HCFMRP/USP e a formar as suas próprias convicções sobre os efeitos de cada etapa desse tratamento. Em muitos casos, os primeiros resultados passam a ser percebidos pela resposta positiva na melhora do quadro clínico e reintegração social, especialmente, no meio familiar. Entretanto, em outros casos, como era de se esperar, esses avanços ainda não se fazem sentir, como se pode apreender dessas descrições:

(...) ela está conseguindo entender as coisas que ela tem, está conseguindo suportar situações que antes ela as tinha como insuportáveis. Sinto uma melhora grande. [P3]

(...) agora ela se abre mais. Quando tem um problema. Já chega, já senta, conversa com a gente. Antes ela não conseguia nem fazer isso. (...) Ela volta diferente. (...) Agora ela voltou a se relacionar. Antes era só a gente, e nem a gente, era mais a cama dela e ela e nem saía de lá. [P5]

(...) por enquanto, doutor, são só essas reuniões aí e não vi efeito. Ela só falou, e não repercutiu em casa. Acho que a parir de agora, com o auxílio da família tudo vai ajudar lá fora. [P9]

(...) os tratamentos aqui no hospital dia servem tanto para o paciente quanto para a família. E já deu uma auxiliada assim, ela está refletindo bastante, porque ela vê os problemas dos outros e vê os problemas dela. [P15]

\section{Grupo Comunitário e Redes Sociais de Apoio}

O Grupo Comunitário é uma das atividades que integra o rol das abordagens terapêuticas institucionais do HD/HCFRP/USP. Com frequência semanal, o grupo é aberto à participação dos pacientes em semi-internação, egressos do HD, familiares e comunidade em geral. Merece destaque o fato de que quando perguntados sobre a realização dessa atividade e o seu funcionamento, apenas um participante, [P8], respondeu conhecê-la, enquanto que todos os demais sequer sabiam da sua existência.

Sobre as Redes Sociais de Apoio as pessoas com transtornos mentais e suas famílias, a maioria dos participantes também referiu desconhecê-las. 
Apenas dois participantes admitiram ter algum conhecimento, mesmo que superficialmente e sem qualquer participação prévia, de atividades dessa natureza em suas comunidades:

(...) lá perto tem. Não no bairro, mas no bairro próximo. Grupo de artesanato, para a comunidade em geral, não só para pacientes com depressão. (...) Não conheço, mas assim: agora eles fizeram uma lista de possíveis lugares que possam fazer esse trabalho. É para a gente ligar e se informar. Eles não sabem se têm todas as atividades, quais as atividades têm, mas me avisaram. [P5] (...) lá tem a prefeitura e a Psicóloga se ela quiser fazer a terapia. É um serviço do município. A gente tem um trabalho de pregação, a gente tá sempre junto na comunidade. O que ela precisa..., o certo seria que cada pessoa ficasse um meio turno com ela. (...) Eu vi outro dia um grupo, uma Psicóloga levando um grupo bem grande. Um grupo de pessoas, que você percebe que são doentes, indo fazer ginástica. [P14]

\section{DISCUSSÃO}

Os transtornos mentais representam um importante problema de saúde pública, interferindo no cotidiano das pessoas, no relacionamento social seja na família, no trabalho ou na comunidade. A Organização Mundial da Saúde - OMS enfatiza que a magnitude do campo da saúde mental pode ser medida pela estimativa mundial de que mais de $25 \%$ das pessoas, independentes da sua localização em país desenvolvido ou em desenvolvimento, apresentam um ou mais transtornos mentais e comportamentais durante o ciclo da vida (Organização Mundial da Saúde, 2001).

Andreoli, Almeida-Filho, Martin, Mateus \& Mari (2007) investigaram o desenvolvimento da infra-estrutura de serviço de saúde mental e do seu financiamento no Brasil e concluíram que existe um claro movimento de transformação do modelo dos cuidados psiquiátricos no Brasil, passando do hospital psiquiátrico para os serviços comunitários. Entretanto, a cobertura dos serviços comunitários ainda é precária e a reforma da assistência psiquiátrica não foi acompanhada pelo aumento do investimento público em saúde mental.

Weber (2012) afirma que para o alcance das medidas que visam a melhoria da atenção à saúde mental, é determinante que as políticas públicas de desospitalização e desinstitucionalização, sugeridas pela Reforma da Assistência Psiquiátrica, sejam acompanhadas de financiamento adequado às necessidades de infraestrutura, expansão e cobertura da rede ambulatorial, o que não vem acontecendo no Brasil, (Andreoli, Almeida-Filho, Martin, Mateus, \& Mari, 2007; Zappitelli, Gonçalves, \& Mosca, 2006) em que pesem algumas exceções que confirmam a regra.

No HD o usuário recebe, diariamente, tratamentos biológicos, psicológicos e sociais executados por uma equipe multidisciplinar. É importante ressaltar o papel da família no tratamento oferecido ao paciente, inclusive a participação nas abordagens terapêuticas que lhe são próprias.

$\mathrm{O}$ arsenal terapêutico para assistir e reabilitar a pessoa com transtorno mental representa tecnologias de cuidado complexas e diversificadas que buscam enfrentar as causas orgânicas, psicológicas e sociais da experiência do adoecimento tanto quanto conhecidas.

Nas estratégias de assistência e cuidado, a responsabilidade cabe não apenas à equipe, mas também ao usuário e à família. Como sujeitos do processo, os pacientes são esclarecidos sobre sua doença e têm autonomia para aderir ou não ao projeto terapêutico proposto (Benevides, Pinto, Cavalcante, \& Jorge, 2010).

As atividades em todos os programas de HD são preponderantemente grupais e quase todos orientados a atividades psicoterápicas, cujo principal objetivo seja promover ao paciente a possibilidade de viver experiências emocionais transformadoras (Bettarello, Silva, Grego, \& Silva Filho, 2008).

Entre as abordagens terapêuticas desenvolvidas no HD/ HCFMRP/USP destaca-se o manejo terapêutico grupal como estratégia de pacientes e familiares no desenvolvimento de habilidades interpessoais importantes como à comunicação mais clara; aproximação com o outro; expressão de sentimentos positivos; atenção e capacidade para escutar; oferecimento de apoio; revelação de si mesmo (Guimarães \& Contel, 2009).

Essas abordagens constituem uma tentativa de compreender o transtorno mental de forma diferente, com ênfase na pessoa com o transtorno, na sua forma de vida, na realidade em que está inserida, e não no transtorno em si, diferentemente da prática constante nos últimos séculos.

Nesse cenário, o HD/HCFMRP/USP desenvolve as suas ações assistenciais clínica e de reabilitação psicossocial dentro de um plano terapêutico monolítico não institucionalizado. O trabalho desenvolvido baseia-se na noção de equipe de trabalho multidisciplinar a partir da abordagem terapêutica fundada teoricamente no Construtivismo Social, na atuação em equipe reflexiva e grupos operativos. 
Entre as abordagens terapêuticas, a psicoeducação ganha espaço como um tratamento adicional ao fármaco, com o propósito de manter o paciente inserido na comunidade. Essa estratégia de cuidado tem como objetivo ajudar o portador a melhorar seu insight sobre a doença, lidar com a estigmatização, melhorar a adesão ao tratamento, ensinar os sinais prodrômicos precoces, promover hábitos saudáveis e a regularidade no estilo de vida e evitar o abuso de substâncias (Miasso, Cassiani, \& Pedrão, 2007).

Rouge \& Aubry (2007) realizaram uma revisão da literatura para investigarem a eficácia da psicoeducação no Transtorno Afetivo Bipolar. Constataram que essa possibilidade terapêutica propicia a melhora na evolução da doença, entre outros fatores, por favorecer o aumento do conhecimento dos pacientes e daqueles que estão envolvidos com a experiência do adoecimento.

Menezes \& Sousa (2012) salientam que em geral, a psicoeducação é considerada como uma das principais estratégias para modificar lembranças negativas vivenciadas pelos portadores de Transtorno Afetivo Bipolar e envolve fornecer informações aos pacientes e familiares sobre a natureza e o tratamento da doença, provendo ensinamentos teóricos e práticos para que possam compreender e lidar melhor com a mesma.

Farina, Terroso, Lopes \& Argimon (2013) realizaram um levantamento acerca de características relevantes que ocorreram ao longo de sessões de psicoeducação em uma comunidade terapêutica para tratamento de dependentes químicos. Os resultados demonstraram que se consubstancia em uma intervenção técnica valiosa nesses tipos de transtornos, pois, tendo como principal proposta educar, o trabalho em grupos possibilita novos conhecimentos sobre as suas demandas e também ajuda diretamente no vínculo entre terapeuta e pacientes, facilitando o entendimento de seus problemas, doenças, ao esclarecer dúvidas e mostrando que os pacientes são ativos na terapia. Foes, Ferreira \& Paludo (2015) relataram uma proposta de intervenção dentro de um Centro de Atenção Psicossocial - Álcool e Drogas, destinada aos usuários de substâncias psicoativas e aos seus familiares. Constataram que a abordagem teórico-prática escolhida como principal estratégia para desenvolvimento da intervenção, a psicoeducação, se mostrou bastante efetiva à troca de informações entre todos os envolvidos nas oficinas, educando o usuário e a sua família, tornando-os ativos nesse processo de lidar com a doença, favorecendo a adesão ao tratamento e reduzindo as chances de recaídas.
As narrativas dos participantes reforçam os resultados e conclusões dos trabalhos revisados e aqui apresentados. Os aprendizados teóricos e práticos acumulados pelas suas participações nas diversas atividades terapêuticas, entre essas, a psicoeducação, permitiram compreender e lidar melhor com o fenômeno do adoecimento e significar os sentimentos vivenciados pela experiência de ser familiar de uma pessoa com transtorno mental.

Com relação ao Grupo Comunitário de Saúde Mental GCSM trata-se de uma iniciativa própria e específica do HD como uma contribuição à comunidade naquilo que se reconhece como rede social de apoio ou suporte social a pessoas com transtornos mentais, no âmbito das abordagens terapêuticas desenvolvidas nessa modalidade de atenção em saúde mental.

Ainda que desconhecido pela maioria dos participantes desta investigação no momento das entrevistas realizadas, impõe-se que se chame a atenção para o fato de que a estruturação do GCSM não se restringe aos limites dos usuários do HD com diagnóstico de patologia psiquiátrica em sua fase aguda ou remissão, em tratamento ou em pós-tratamento. É um grupo mais amplo e aberto à comunidade.

A PNSM ao redirecionar a assistência psiquiátrica adotou um modelo em rede de atenção psicossocial que favoreça a integração social e familiar das pessoas com transtornos mentais. Weber \& Juruena (2016) salientam que o hospital dia em saúde mental ao tratar o paciente durante um tempo limitado sem a necessidade de afastamento do seu meio social, familiar, de trabalho ou acadêmico, tenta prevenir a reclusão e a marginalização/exclusão do paciente psiquiátrico.

Nesse cenário é importante se chamar à atenção a duas questões. A primeira é aquela que se refere ao fato de que o transtorno mental constituiu desde sempre um desafio à capacidade de as sociedades integrarem e cuidarem dos seus portadores (Hespanha, 2010).

A segunda, é que uma das maiores preocupações da Psiquiatria deve ser auxiliar pessoas a libertarem-se das limitações impostas pelos quadros clínicos do transtorno ou, quando isso não é possível, a melhor conviverem com suas deficiências (Brusamarello, Guimarães, Labronici, Mazza, \& Maftum, 2011).

Assim, o cuidado em saúde mental tem evidenciado a necessidade de focar a atenção para intervenções que ofereçam alternativas de se trabalhar a realidade social, a fim de promover suporte mútuo, democracia participativa e movimentos sociais. 
Em linhas gerais, os Programas de Suporte Comunitário - PSC consistem numa rede organizada de cuidados, composta por pessoas da comunidade, que se comprometem a prestar assistência à população com transtornos mentais assistindo suas necessidades, a fim de reduzir as hospitalizações e favorecer a evolução clínica, o funcionamento social e a qualidade de vida, sem que o paciente seja isolado da comunidade (Brusamarello, Guimarães, Labronici, Mazza, \& Maftum, 2011).

A noção de rede social de apoio simbolizada como um conjunto de pontos conectados por fios, de modo a formar a imagem de uma teia representa, comumente, a mobilização comunitária no propósito de contribuir no enfrentamento dos problemas de saúde vivenciados por integrantes do seu meio social.

A presença de uma doença ou incapacitação crônica não só provoca a erosão na rede social habitual como, às vezes, também pode gerar novas redes como as que correspondem aos serviços sociais, de saúde ou grupos terapêuticos.

No campo da saúde mental, a intervenção em redes sociais tem apresentado crescimento significativo nas estratégias de cuidado e, especialmente, nas práticas desenvolvidas pelos serviços substitutivos territoriais (Muramoto, 2008).

Entretanto, o retorno à vida comunitária de pessoas com transtornos mentais não vem se mostrando uma tarefa fácil por diversas razões, entre as quais, o fato de que os locais onde está sendo realizada a assistência, seja em regime de internação integral, semi-internação ou em Centros de Atenção Psicossocial, como exemplos, acabam por se transformar no único centro de referência, proteção e aceitação.

Como questão a ser explorada, resta saber como as propostas alternativas em Saúde Mental pensam em preservar a possibilidade de uma temporalidade diferenciada, em que a lentidão não seja impotência, em que a diferença de ritmos não seja disritmia, em que os movimentos não ganhem sentidos apenas pelo seu desfecho.

De um lado, os meios terapêuticos devem propiciar, passo a passo, condições para a aplicação das intervenções terapêuticas necessárias à reabilitação psicossocial da pessoa com transtorno mental. Esse trabalho será muito facilitado com apoio da família e a criação de dispositivos sociais que tornem menos problemático o engajamento do paciente na comunidade.
De outro, e concordante com diversos autores, a realidade de que a mobilização sistemática das estruturas sociais para favorecer esse trabalho de reabilitação é praticamente nula, dificulta muito a construção de caminhos que possibilitem ao paciente psiquiátrico crônico participar ativamente da sociedade, mesmo nos casos em que sua contribuição poderia ser extremamente profícua (Brusamarello, Guimarães, Labronici, Mazza, \& Maftum, 2011).

Por tal razão, encaminhar o paciente para uma instituição de ensino, de trabalho ou de amparo social sem o cuidadoso preparo poderia comprometer todo o sucesso terapêutico alcançado anteriormente, especialmente por desconhecer que poucos são os recursos sociais disponíveis em nosso meio para suprir as necessidades de pacientes com limitações de funcionamento psicossocial (Brusamarello, Guimarães, Labronici, Mazza, \& Maftum, 2011).

Sem descuido, o suporte teórico e prático propiciado pelas atividades de grupo e psicoeducação torna possível a pacientes e familiares o esclarecimento de suas dúvidas e a superação dos medos e mitos que envolvem o transtorno mental. A melhoria na qualidade de vida secundária ao reforço da auto-estima e do bem-estar facilita o lidar com a vivência do adoecimento nas limitações das atividades de vida diária, na sobrecarga do cuidado e nas rupturas das relações sociais resultantes do estigma e do preconceito associados às pessoas com transtornos mentais (Tursi-Braga, 2014).

As pesquisas brasileiras sobre a inclusão social de pessoas com transtornos mentais em tratamento em hospital dia, sobretudo, se considerado o tema a partir do entendimento da família se mostra insuficiente como revela o estudo de Weber \& Juruena (2014). Na esteira dessa constatação novas investigações, entre as quais, estudos de seguimento de pessoas com transtornos mentais que participam de serviços de saúde mental ambulatoriais de base comunitária, de grupos comunitários e de outras modalidades terapêuticas que integram a rede social de apoio devem ser estimulados para confrontar as práticas de cuidado adotadas com o desfecho clínico e social desejados.

Por fim, é importante ressaltar, para que não se perca de vista, as implicações para prática clínica advindas de pesquisas e de serviços que considerem a maneira pela qual a família de pacientes com transtornos mentais não só compreendem o transtorno mental de seu familiar, mas, sobretudo como significam este transtorno e as terapêuticas adotadas pelos equipamentos de saúde mental responsáveis pelos cuidados dos mesmos. 
Os serviços substitutivos em saúde mental como, por exemplo, o hospital dia, deve estar alerta no auxilio as famílias envolvidas neste fenômeno muito peculiar de adoecimento, de modo a estar atento à escuta das dificuldades e dúvidas trazidas pelas famílias para o enfrentamento de tal experiência. Ajudá-las na compreensão do transtorno mental, dos seus sintomas e, sobretudo, na complexidade que circunscreve tal enfermidade eleva o serviço de saúde à condição de elemento facilitador para o alcance de um manejo mais qualificado por parte da família.

Desse modo, intervenções nessa direção propiciam um melhor relacionamento familiar como também reforçam o vínculo entre o serviço de saúde, paciente e família impactando positivamente os indicadores de saúde, especialmente na redução das taxas de abandono de tratamento e de re-internações, o que aumentam as chances de êxito no propósito de inserção social, à medida que as atividades de vida diária, de relação social e laboral possam ser recuperadas.

\section{CONCLUSÕES}

A psicoeducação é uma modalidade de intervenção psicossocial que vem se consolidando como estratégia para informar, pacientes e familiares, sobre o diagnóstico, terapêuticas e prognóstico da doença reforçando a necessidade de adesão ao tratamento e da conscientização para um estilo de vida com hábitos saudáveis.

O GCSM constitui-se em uma estratégia de cuidado do plano terapêutico, estendendo-se como uma atividade de apoio social comunitário na medida em que permite a participação de usuários de outros serviços de saúde mental e a comunidade em geral. A valorização crescente dessa experiência permitiu a ampliação dos temas abordados para além da terapêutica (clínica, psicofarmacológica e psicoterápica), consolidando a proposta como uma busca por saúde mental.

Entretanto, observa-se que tanto os efeitos da hospitalização quanto a carência de iniciativas para compensar a falta de uma rede de suporte social e comunitário à pacientes psiquiátricos, agravam ainda mais as discriminações sofridas, pelo afastamento da sociedade e dos familiares.

O desafio nessas situações é aquele em que se constata na incapacidade ou impossibilidade de fazer a "ponte" entre as abordagens terapêuticas desenvolvidas nesses serviços de saúde mental e a comunidade. No primeiro caso, a incapacidade pode estar na razão direta das limitações impostas pela própria doença mental.
No segundo caso, a impossibilidade se materializa pela inexistência de suporte social comunitário nos moldes experimentados durante o tratamento especializado. Todavia, no percurso terapêutico do HD/HCFMRP/ USP a família observa, gradativamente, os resultados positivos das intervenções propostas, reconhecendo que tais atividades, clínicas e psicossociais, são medidas que contribuem para a integração quando estes retornam para casa.

\section{REFERÊNCIAS BIBLIOGRÁFICAS}

Andreoli, S. B., Almeida-Filho, N., Martin, D., Mateus, M. D. M. L., \& Mari, J. J. (2007). Is psychiatric reform a strategy for reducing the mental health budget? The case of Brazil. Revista Brasileira de Psiquiatria, 29(1), 43-46. doi: 10.1590/S1516-44462006005000032

Benevides, D. S., Pinto, A. G. A., Cavalcante, C. M., \& Jorge, M. S. B. (2010). Cuidado em saúde mental por meio de grupos terapêuticos de um hospital dia: Perspectivas dos trabalhadores de saúde. Interface (Botucatu), 14(32), 127-138. doi: 10.1590/S141432832010000100011

Bettarello, S. V., Silva, M. C. F., Grego, F., \& Silva Filho, L. M. A. (2008). Fundamentos e prática em hospital dia e reabilitação psicossocial. São Paulo: Atheneu.

Brusamarello, T., Guimarães, A. N., Labronici, L. M., Mazza, V. A., \& Maftum, M. A. (2011). Redes sociais de apoio de pessoas com transtornos mentais e familiares. Texto \& Contexto Enfermagem, 20(1), 33-40. doi: 10.1590/S0104-07072011000100004

Farina, M., Terroso, L. B., Lopes, R. M. F., \& Argimon, I. (2013). Importância da psicoeducação em grupos de dependentes químicos: Relato de experiência. Aletheia, 42, 175-185.

Foes, V. F. L., Ferreira, L. S., \& Paludo, S. S. (2015). Caindo na real: Relato de uma experiência de psicoeducação no tratamento da dependência química. Revista Saúde e Desenvolvimento Humano, 3(1), 61-70.

Guimaraes, A. C. P. C., \& Contel, J. O. B. (2009). Psicoterapia de grupo em hospital dia psiquiátrico. Paidéia, 19(44), 379-385. doi: 10.1590/S0103863X2009000300012 
